Brief paper

\title{
Boundary second-order sliding-mode control of an uncertain heat process with unbounded matched perturbation ${ }^{\text {* }}$
}

\author{
Alessandro Pisano ${ }^{1}$, Yury Orlov ${ }^{2}$ \\ Department of Electrical and Electronic Engineering, University of Cagliari, Cagliari, Italy
}

\section{A R T I C L E I N F O}

\section{Article history:}

Received 26 May 2010

Received in revised form

11 October 2011

Accepted 23 January 2012

Available online 22 June 2012

\section{Keywords:}

Infinite-dimensional systems

Boundary control

Heat equation

Uncertain systems

Second-order sliding modes

\begin{abstract}
A B S T R A C T
The primary concern of the present paper is the regulation of an uncertain heat process with collocated boundary sensing and actuation. The underlying heat process is governed by an uncertain parabolic partial differential equation (PDE) with Neumann boundary conditions. It exhibits an unknown constant diffusivity parameter and it is affected by a smooth boundary disturbance, which is not available for measurements and which is possibly unbounded in magnitude. The proposed robust synthesis is formed by the linear feedback design and by the "Twisting" second-order sliding-mode control algorithm, suitably combined and re-worked in the infinite-dimensional setting. A non-standard Lyapunov functional is invoked to establish the global asymptotic stability in a Sobolev space, involving spatial state derivatives of the same order as that of the plant equation. The stability proof is accompanied by a set of simple tuning rules for the controller parameters. The effectiveness of the developed control scheme is supported by simulation results.
\end{abstract}

(C) 2012 Elsevier Ltd. All rights reserved.

\section{Introduction}

Sliding-mode control has long been recognized as a powerful control method to counteract non-vanishing external disturbances and unmodeled dynamics (Utkin, 1983). This method is based on the deliberate introduction of sliding motions into the control system, and, since the motion along the sliding manifold proves to be uncorrupted by matched disturbances, the closed-loop system is guaranteed to exhibit strong properties of robustness against significant classes of disturbances and model uncertainties. Due to these advantages and the simplicity of implementation, slidingmode controllers have widely been used in various applications (Orlov, 2009).

On the other hand, many important engineering systems and industrial processes are governed by partial differential equations (PDEs) and are often subject to a significant degree of uncertainty.

\footnotetext{
is Y. Orlov acknowledges the financial support of the University of Cagliari and Region of Sardinia in the framework of the 2009/2010 "Long Stay" Visiting Professor Program. A. Pisano gratefully acknowledges the financial support from the European Union Seventh Framework Programme (FP7/2007-2013) Network of excellence "HYCON2-Highly-complex and networked control systems" under grant agreement n. 257462. The material in this paper was not presented at any conference. This paper was recommended for publication in revised form by Associate Editor Nicolas Petit under the direction of Editor Miroslav Krstic.

E-mail addresses: pisano@diee.unica.it (A. Pisano), yorlov@cicese.mx (Y. Orlov). 1 Tel.: +39 0706755760; fax: +390706755782.

2 On sabbatical leave from CICESE Research Center, Electronics and Telecommunication Department, Ensenada, Mexico.
}

Therefore, a growing interest is arising towards extending the discontinuous control methods to infinite-dimensional systems. Presently, the discontinuous control synthesis in the infinitedimensional setting is well documented (Levaggi, 2002; Orlov, 2000; Orlov, Liu, \& Christofides, 2004; Orlov \& Utkin, 1987) and it is generally shown to retain the main robustness features as those possessed by its finite-dimensional counterpart. Other robust control paradigms have been fruitfully applied as well in the infinite dimensional setting such as adaptive control (Krstic \& Smyshlyaev, 2008b), and LMI-based design (Fridman \& Orlov, 2009). The recent books (Christofides, 2001; Orlov, 2009) illustrate some existing results in the field of robust control of infinite dimensional systems.

The boundary control problem for heat processes was studied, e.g., in Boskovic, Krstic, and Liu (2001), Fridman and Orlov (2009) and Krstic and Smyshlyaev (2008a) under more strict assumptions on the admitted uncertainties and perturbations compared to those made in the present work. In this paper we address the boundary control problem for an uncertain heat process, governed by a parabolic PDE with a scalar spatial variable $\xi \in[0,1]$ and with Neumann boundary conditions (BC's). An appropriate extension of second-order sliding mode (2-SM) control techniques (Levant, 1993; Orlov, 2009) allows us to address the following main features:

- The diffusivity parameter is admitted to be an unknown positive constant.

- Only collocated boundary sensing and actuation are assumed to be available. 
- The proposed dynamic controller rejects a class of locally integrable matched disturbances of an arbitrary shape. Such a disturbance is admitted to be non-vanishing and unbounded in magnitude whereas the norm of its time derivative is assumed to be uniformly bounded by an a priori known constant.

- The plant input is continuous, whereas its first-order time derivative is composed of a discontinuous part (implementing the "Twisting" 2-SM algorithm Levant, 1993) and a continuous linear part.

- The global asymptotic stability of the closed-loop system is achieved in an appropriate Sobolev space.

The combined use of the Twisting 2-SM and linear feedback was already suggested in Orlov (2009), and the main novelty here is the application of this algorithm to regulate an infinite dimensional system through its boundary. In the resulting closedloop system, the controller is connected to the plant through a dynamical filter (an integrator) thereby augmenting the system state with its time derivative. While passing through the integrator, the discontinuous signal is smoothed out, and the so-called chattering phenomenon, extremely undesired in practice, is thus attenuated. Due to such a dynamic input extension, the global asymptotic stabilization of the underlying uncertain heat process is achieved in a stronger norm of a Sobolev space, involving spatial state derivatives up to the second order. This constitutes the main contribution of the paper. The stability proof is based on a non-standard Lyapunov functional construction, invented for the proposed closed-loop system. The proof leads to a set of simple tuning rules for the controller parameters.

Some related works (Orlov, Pisano, \& Usai, 2010, 2011a,b) have recently been published by the authors. In those works natural generalizations of the Twisting and Supertwisting 2-SM algorithms to the infinite-dimensional setting were developed to address the tracking control of uncertain and perturbed heat and wave propagation processes. In the aforementioned works, however, the available sensing and actuation were assumed to be distributed over the entire state spatial domain, whereas the present development makes a fundamental step beyond that assumption by admitting only boundary sensing and actuation to be available.

The rest of the paper is outlined as follows. In Section 2 , a family of perturbed heat equations is introduced and the control problem is formulated. In Section 3, a stabilizing boundary controller is developed and appropriate solution concepts are specified. In Section 4 the main result and the associated stability proof are presented. Simulation results are given in Section 5. Finally, Section 6 collects some concluding remarks.

\subsection{Notation}

The notation used throughout is fairly standard. $H^{l}(0,1)$, with $l=0,1,2, \ldots$, denotes the Sobolev space of absolutely continuous scalar functions $z(\zeta)$ on $(0,1)$ with square integrable derivatives $z^{(i)}(\zeta)$ up to the order $l$ and the $H^{l}$-norm

$\|z(\cdot)\|_{l}=\sqrt{\int_{0}^{1} \Sigma_{i=0}^{l}\left[z^{(i)}(\zeta)\right]^{2} d \zeta}$.

Throughout the paper we shall also utilize the standard notation $H^{0}(0,1)=L^{2}(0.1)$.

\section{Problem formulation}

Consider the space- and time-varying scalar field $Q(\xi, t)$ with the monodimensional (1D) spatial variable $\xi \in[0,1]$ and time variable $t \geq 0$. Let it be governed by a parabolic PDE which is commonly referred to as the "Heat Equation":

$Q_{t}(\xi, t)=\theta Q_{\xi \xi}(\xi, t)$,

where $Q_{t}$ and $Q_{\xi \xi}$ denote temporal and second-order spatial derivatives, respectively, and $\theta$ is a positive unknown coefficient called thermal conductivity (or, more generally, diffusivity). The initial condition (IC) is

$Q(\xi, 0)=Q^{0}(\xi) \in H^{4}(0,1)$.

Throughout, we consider controlled and perturbed Neumanntype BC's of the form

$Q_{\xi}(0, t)=0, \quad Q_{\xi}(1, t)=u(t)+\psi(t)$,

where $u(t) \in \mathbb{R}$ is a modifiable source term (boundary control input) and $\psi(t) \in \mathbb{R}$ represents an uncertain sufficiently smooth disturbance. The class of initial functions and admissible disturbances is specified by the following assumption.

Assumption 1. The initial function $Q^{0}(\xi)$ in the ICs (3) is compatible to the next perturbed BC's

$Q_{\xi}^{0}(0)=0, \quad Q_{\xi}^{0}(1)=\psi(0)$,

whereas the disturbance $\psi(t)$ is twice continuously differentiable, and there exists an a priori known constant $M>0$ such that

$\left|\psi_{t}(t)\right| \leq M, \quad \forall t \geq 0$

It is worth noting that $\mathrm{BC}(4)$ at the boundary $\xi=1$ considered along with (5) implies that $u(0)=0$. With the assumption above, the stability of the considered heat process is studied in an appropriate Sobolev space being specified to $H^{2}(0,1)$. By virtue of this, the domain $D(\mathcal{A})$ of the infinitesimal operator $\mathcal{A}=\frac{\partial^{2}}{\partial \xi^{2}}$ in the boundary value problem (2)-(4) is confined to the Sobolev space $H^{4}(0,1)$ and the corresponding boundary conditions (4).

The control objective is to steer the $H^{2}$-norm $\|x(\cdot, t)\|_{2}$ of the deviation

$x(\xi, t)=Q(\xi, t)-Q^{r}$

of the scalar field $Q(\xi, t)$ from an a priori given time-independent and spatially-invariant reference $Q^{r}$ to zero, despite the presence of an uncertain boundary disturbance $\psi(t)$, arbitrarily shaped and possibly unbounded in magnitude. Boundary sensing at $\xi=1$ of the deviation $x(\xi, t)$ and of its time derivative $x_{t}(\xi, t)$ is assumed to be the only available information on the state of the system. The deviation variable $x(\xi, t)$ is governed by the heat equation

$x_{t}(\xi, t)=\theta x_{\xi \xi}(\xi, t)$

subject to the same Neumann-type BC's

$x_{\xi}(0, t)=0, \quad x_{\xi}(1, t)=u(t)+\psi(t)$,

and IC's

$x(\xi, 0)=x^{0}(\xi), \quad x^{0}(\xi)=Q^{0}(\xi)-Q^{r}$.

It should be noted that according to Curtain and Zwart (1995, Theorem 3.3.3), the unforced system (8)-(10) with $u(t) \equiv 0$ and under Assumption 1 possesses a unique classical solution that by definition (see Curtain \& Zwart, 1995, Definition 3.2.9) meets the boundary-value problem point-wise. 


\section{Stabilizing synthesis}

To achieve the control goal, the system state is augmented through a dynamic input extension by inserting an integrator at the plant input. The control derivative $u_{t}(t)$ is then regarded as a fictitious control variable to be generated by a suitable feedback mechanism.

The following dynamic controller

$$
\begin{aligned}
\dot{u}(t)= & -\lambda_{1} \operatorname{sign} x(1, t)-\lambda_{2} \operatorname{sign} x_{t}(1, t)-W_{1} x(1, t) \\
& -W_{2} x_{t}(1, t), \quad u(0)=0,
\end{aligned}
$$

is currently under study, where, according to (5), the initial value $u(0)$ is set to zero to verify the compatibility ${ }^{3} x_{\xi}^{0}(1)=u(0)+\psi(0)$ to the BC's (9). In the above relation, $\lambda_{1}, \lambda_{2}, W_{1}$ and $W_{2}$ are constant parameters subject to the inequalities

$\lambda_{2}>M, \quad \lambda_{1}>\lambda_{2}+M, \quad W_{1}>0, \quad W_{2}>0$,

and sign $\cdot$ stands for the multi-valued function $\operatorname{sign} z: \mathbb{R} \rightarrow$ $[-1,1]$ such that

$\operatorname{sign} z \in\left\{\begin{array}{cc}1 & z>0 \\ {[-1,1]} & z=0 \\ -1 & z<0\end{array}\right.$

The proposed boundary dynamic controller (11) is composed of the so-called twisting controller (Levant, 1993), governed by the first two commuting terms, and the linear part, being a PD controller. It makes explicit use of $Q(1, t)$ and $Q_{t}(1, t)$ for feedback. Despite the state derivative is normally not permitted to use in the synthesis (as it generally induces algebraic loops) its use becomes acceptable when a dynamic input extension is performed, similar to that of the present paper where the input signal passes through an integrator and, by virtue of this, the system state is augmented by $Q_{t}$ being viewed as a component of the augmented state vector $\left(Q, Q_{t}\right)$. As a consequence, our stability result (Theorem 1 ), formulated below, not only ensures the asymptotic stability of $x(\xi, t)$ in $H^{2}$-norm but due to (8) it also ensures asymptotic stability of $x_{t}(\cdot, t)$ in $L^{2}$-norm.

Since the dynamic control input is governed by the ordinary differential equation (11) with discontinuous (multi-valued) righthand side, the precise meaning of the solutions of the distributed parameter system (8)-(11), driven by the discontinuous dynamic controller (11), is then specified in the sense of Filippov (1988). Extension of the Filippov concept towards the infinite-dimensional setting may be found in Levaggi (2002) and Orlov (2009). As in the finite-dimensional case, a motion along the discontinuity manifold, if any, is referred to as a sliding mode.

It is well-known from the finite-dimensional theory (Orlov, 2005) that the combined twisting-PD algorithm (11) generates a sliding mode on the discontinuity manifolds intersection $x(1, t)=$ $x_{t}(1, t)=0$ only. Thus, beyond this intersection, state trajectories always cross the discontinuity manifolds $x(1, t)=0$ and $x_{t}(1, t)=$ 0 , and therefore, they constitute Caratheodory solutions, i.e., the state equations are verified for almost all time instants everywhere except the discontinuity manifolds intersection where the closedloop system stays in equilibrium to be viewed in the sense of Filippov.

Because of space limitations the present paper focuses on the stabilizing synthesis whereas the rigorous demonstration of the well-posedness of the closed-loop system (8)-(10), (11) remains an open problem which is beyond the scope of the paper. The well-posedness of the system in question under the assumptions,

\footnotetext{
3 See, e.g., Vazquez and Krstic (2007) for the need of certain compatibility conditions in the dynamic boundary control synthesis.
}

imposed on the IC's and BC's, is actually verified in accordance with (Curtain \& Zwart, 1995, Theorem 3.3.3) by taking into account that the control input $u(t)$, governed by the combined twisting algorithm (11), is twice piece-wise continuously differentiable along the state trajectories.

Thus, in the remainder, we simply assume the following.

Assumption 2. The closed-loop system (8)-(11) possesses a unique Filippov solution $x(\cdot, t) \in H^{4}(0,1)$ and its time derivative $x_{t}(\cdot, t)=z(\cdot, t) \in H^{2}(0,1)$ verifies the following auxiliary boundary-value problem

$z_{t}(\xi, t)=\theta z_{\xi \xi}(\xi, t)$,

$z_{\xi}(0, t)=0, \quad z_{\xi}(1, t)=\dot{u}(t)+\dot{\psi}(t)$,

$z(\xi, 0)=z^{0}(\xi)$,

where

$z^{0}(\xi)=\theta x_{\xi \xi}^{0}(\xi) \in H^{2}(0,1)$.

Notice that the auxiliary problem (14)-(16) is formally obtained by differentiating (8)-(10) in the time variable whereas the IC (17) is straightforwardly derived from (8), (10).

It should be noted that even the existence of classical solutions of the unforced auxiliary boundary-value problem $(14)-(16)$ is no longer guaranteed because Curtain and Zwart (1995, Theorem 3.3.3) proves to be inapplicable to the system in question, perturbed with a function $\dot{\psi}$ of class $C^{1}$, and which in addition does not necessarily verify the resulting compatibility condition $x_{\xi \xi}^{0}(1)=\dot{\psi}(0)$. Moreover, the closed-loop version $x_{\xi \xi}^{0}(1)=$ $\dot{u}(0)+\dot{\psi}(0)$ of the compatibility condition becomes exotic under the control algorithm (11) according to which $\dot{u}(0)=$ $-\lambda_{1} \operatorname{sign} x(1,0)-\lambda_{2} \operatorname{sign} x_{t}(1,0)-W_{1} x(1,0)-W_{2} x_{t}(1,0)$. Thus, it seems unacceptable to invoke such a compatibility condition in the closed-loop setting, which is why the meaning of the auxiliary boundary-value problem (14)-(16), (11), is subsequently viewed in the mild sense (see, e.g., Curtain \& Zwart, 1995 for details).

Let us recall (Butkovskiy, 1982) that the mild solutions of (14)(16), (11) coincide with the corresponding weak solutions of the so-called standardizing PDE in distributions

$z_{t}(\xi, t)=\theta z_{\xi \xi}(\xi, t)+\theta[\dot{u}(t)+\dot{\psi}(t)] \delta(\xi-1)$,

subject to the homogeneous BCs

$z_{\xi}(0, t)=0, \quad z_{\xi}(1, t)=0$,

and to the same ICs (16).

Since the right-hand side of (18) contains the Dirac distribution $\delta(\xi-1)$, the meaning of (18) is defined indirectly according to the following (see Pazy, 1992, p. 258).

Definition 1. A continuous function $z(\cdot, t) \in L^{2}(0,1)$, satisfying the BC's (19), is said to be a weak solution of the boundary value problem (18)-(19) on $[0, \tau)$ if for every $\varphi(\xi) \in C^{2}(0,1)$ such that $\varphi_{\xi}(0)=\varphi_{\xi}(1)=0$, the function $\int_{0}^{1} z(\xi, t) \varphi(\xi) d \xi$ is absolutely continuous on $[0, \tau)$ and relation

$$
\begin{aligned}
\frac{d}{d t} \int_{0}^{1} z(\xi, t) \varphi(\xi) d \xi= & \theta \int_{0}^{1} z(\xi, t) \varphi_{\xi \xi}(\xi) d \xi \\
& +\theta[\dot{u}(t)+\dot{\psi}(t)] \varphi(1)
\end{aligned}
$$

holds for almost all $t \in[0, \tau)$.

In (20), the Dirac function definition $\int_{0}^{1} \varphi(\xi) \delta(\xi-1) d \xi=\varphi(1)$ and the property $\int_{0}^{1} z_{\xi \xi}(\xi, t) \varphi(\xi) d \xi=\int_{0}^{1} z(\xi, t) \varphi_{\xi \xi}(\xi) d \xi$ of the Sobolev derivative $z_{\xi \xi}(\xi, t)$ under BC's (19) have been taken into account. 


\section{Main result}

We are now in a position to state our main result.

Theorem 1. Consider the perturbed heat process (2)-(4) with Assumptions 1 and 2 above and with the dynamic control strategy (11)-(12) applied. Then the solutions $x(\cdot, t)$ of the resulting error boundary-value problem (8)-(11) are globally asymptotically stable in the space $H^{2}(0,1)$.

The proof of the main result is preceded by few technical lemmas that will be instrumental in our further derivation.

\subsection{Instrumental lemmas}

Lemma 1. Let $y(\xi) \in H^{1}(0,1)$. Then, the following inequality holds:

$\|y(\cdot)\|_{0}^{2} \leq 2\left(y^{2}(i)+\left\|y_{\xi}(\cdot)\right\|_{0}^{2}\right), \quad i=0,1$.

Proof of Lemma 1. Given $y(\xi) \in H^{1}(0,1)$, it is absolutely continuous and therefore,

$y(\xi)=y(0)+\int_{0}^{\xi} y_{\xi}(\eta) d \eta, \quad \forall \xi \in[0,1]$.

Now squaring both sides of (22) and applying the well-known inequality $2 a b<a^{2}+b^{2}$ yield (21) with $i=0$. The proof of (21) with $i=1$ becomes identical under the change of coordinate $\zeta=1-\xi$. Lemma 1 is proved.

Lemma 2. The functional

$\tilde{V}\left(x, x_{t}\right)=\lambda_{1} \theta|x(1, t)|+\frac{1}{2} \theta W_{1} x^{2}(1, t)+\frac{1}{2}\left\|x_{t}(\cdot, t)\right\|_{0}^{2}$,

being computed on the solutions $x(\cdot, t)$ of the boundary-value problem (8)-(11), is equivalent to the $H^{2}$-norm of these solutions in the sense that

$$
\begin{aligned}
& \alpha\left(\|x(\cdot, t)\|_{2}^{2}+\left\|x_{t}(\cdot, t)\right\|_{0}^{2}\right) \\
& \quad \leq \tilde{V}\left(x, x_{t}\right) \leq \beta\left(\|x(\cdot, t)\|_{2}+\|x(\cdot, t)\|_{2}^{2}+\left\|x_{t}(\cdot, t)\right\|_{0}^{2}\right)
\end{aligned}
$$

for an arbitrary solution $x(\cdot, t)$ of (8)-(11), for all $t \geq 0$, and for some positive constants $\alpha, \beta$.

Proof of Lemma 2. Successively applying relation (21) first with $i=1$ to a solution $x(\xi, t)$ of (8)-(11) and then, with $i=0$, to its spatial derivative $x_{\xi}(\xi, t)$ yields

$\|x(\cdot, t)\|_{0}^{2} \leq 2\left(x^{2}(1, t)+\left\|x_{\xi}(\cdot, t)\right\|_{0}^{2}\right)$,

$\left\|x_{\xi}(\cdot, t)\right\|_{0}^{2} \leq 2\left(x_{\xi}^{2}(0, t)+\left\|x_{\xi \xi}(\cdot, t)\right\|_{0}^{2}\right)$.

Coupled to the $\mathrm{BC}(9)$ at $\xi=0$, relation (26) takes the form

$\left\|x_{\xi}(\cdot, t)\right\|_{0}^{2} \leq 2\left\|x_{\xi \xi}(\cdot, t)\right\|_{0}^{2}$

and by taking into account (25), it follows that

$\|x(\cdot, t)\|_{0}^{2} \leq 2\left(x^{2}(1, t)+2\left\|x_{\xi \xi}(\cdot, t)\right\|_{0}^{2}\right)$.

Applying (27), (28) to the norm definition

$\|x(\cdot, t)\|_{2}^{2}=\|x(\cdot, t)\|_{0}^{2}+\left\|x_{\xi}(\cdot, t)\right\|_{0}^{2}+\left\|x_{\xi \xi}(\cdot, t)\right\|_{0}^{2}$,

and utilizing relation $\left\|x_{t}(\cdot, t)\right\|_{0}=\theta\left\|x_{\xi \xi}(\cdot, t)\right\|_{0}$, which is guaranteed by (8), one arrives at

$$
\begin{aligned}
\|x(\cdot, t)\|_{2}^{2} & \leq 2 x^{2}(1, t)+7\left\|x_{\xi \xi}(\cdot, t)\right\|_{0}^{2} \\
& =2 x^{2}(1, t)+\frac{7}{\theta^{2}}\left\|x_{t}(\cdot, t)\right\|_{0}^{2} .
\end{aligned}
$$

Combining (23) and (30), the validity of the first inequality of (24) is then concluded for all $t \geq 0$ and for some positive $\alpha$.

To reproduce the second inequality of (24), let us note that by virtue of the $\mathrm{BC}(9)$ at $\xi=0$, the following chain of inequalities

$$
\begin{aligned}
x_{\xi}^{2}(\xi, t) & =\left(\int_{0}^{\xi} x_{\zeta \zeta}(\zeta, t) d \zeta\right)^{2} \leq \int_{0}^{\xi} x_{\zeta \zeta}^{2}(\zeta, t) d \zeta \\
& \leq\left\|x_{\xi \xi}(\cdot, t)\right\|_{0}^{2}
\end{aligned}
$$

holds for all $\xi \in[0,1]$ and all $t \geq 0$. In turn, employing (31), one obtains

$$
\begin{aligned}
x^{2}(1, t) & =\int_{0}^{1}[x(1, t)-x(\zeta, t)+x(\zeta, t)]^{2} d \zeta \\
& \leq \frac{1}{2}\left\{\int_{0}^{1}[x(1, t)-x(\zeta, t)]^{2} d \zeta+\int_{0}^{1} x^{2}(\zeta, t) d \zeta\right\} \\
& \leq \frac{1}{2}\left\{\int_{0}^{1} \max _{\zeta \in[0,1]} x_{\zeta}^{2}(\zeta, t) d \zeta+\|x(\cdot, t)\|_{0}^{2}\right\} \\
& \leq \frac{1}{2}\left\{\left\|x_{\xi \xi}(\cdot, t)\right\|_{0}^{2}+\|x(\cdot, t)\|_{0}^{2}\right\} \leq \frac{1}{2}\|x(\cdot, t)\|_{2}^{2} .
\end{aligned}
$$

To complete the proof it remains to note that the second inequality of (24) is guaranteed by (32). Lemma 2 is proved.

\section{Lemma 3. Let a set}

$\mathcal{D}_{R}^{\tilde{V}}=\left\{(p(\xi), h(\xi)) \in H^{2}(0,1) \times L^{2}(0,1): \tilde{V}(p, h) \leq R\right\}$

be determined by means of functional (23) and be specified with some positive $R$. Then the following conditions

$p(1) \int_{0}^{1} h(\eta) d \eta \geq-\frac{1}{2}\left[\frac{R}{\lambda_{1} \theta}|p(1)|+\|h\|_{0}^{2}\right]$,

$\|h\|_{0}^{2} \leq 2 R, \quad\|h\|_{0} \leq \sqrt{2 R}, \quad\|h\|_{0}^{2} \leq \sqrt{2 R}\|h\|_{0}$,

hold for an arbitrary $(p(\xi), h(\xi)) \in \mathcal{D}_{R}^{\tilde{V}}$.

Proof of Lemma 3. The following implications hold:

$\tilde{V}(p, h)=\theta \lambda_{1}|p(1)|+\frac{1}{2} \theta W_{1} p^{2}(1)+\frac{1}{2}\|h\|_{0}^{2} \leq R$

$\Rightarrow \theta \lambda_{1}|p(1)| \leq R \Rightarrow|p(1)| \leq \frac{R}{\lambda_{1} \theta}$.

Furthermore, applying the well-known inequality $a b \geq-\frac{1}{2}\left(a^{2}+\right.$ $b^{2}$ ) yields

$$
\begin{aligned}
\int_{0}^{1} p(1) h(\xi) d \xi & \geq-\frac{1}{2}\left[p^{2}(1)+\|h\|_{0}^{2}\right] \\
& =-\frac{1}{2}\left[|p(1)||p(1)|+\|h\|_{0}^{2}\right] .
\end{aligned}
$$

Being coupled together, (35) and (37) immediately result in (34). In turn, the relations (35) follow from the trivial chain of implications:

$\tilde{V}(p, h)=\theta \lambda_{1}|p(1)|+\frac{1}{2} \theta W_{1} p^{2}(1)+\frac{1}{2}\|h\|_{0}^{2} \leq R$

$\Rightarrow \frac{1}{2}\|h\|_{0}^{2} \leq R \Rightarrow\|h\|_{0} \leq \sqrt{2 R}$

$\Rightarrow\|h\|_{0}^{2} \leq \sqrt{2 R}\|h\|_{0}$.

Lemma 3 is thus proved. 


\subsection{Proof of Theorem 1}

By Lemma 2, functional (23) is positive definite on the solutions $x(\cdot, t)$ of the boundary-value problem (8)-(11) and its time derivative is given by

$$
\begin{aligned}
\dot{\tilde{V}}(t)= & \lambda_{1} \theta x_{t}(1, t) \operatorname{sign}(x(1, t))+W_{1} \theta x(1, t) x_{t}(1, t) \\
& +\int_{0}^{1} x_{t}(\eta, t) x_{t t}(\eta, t) d \eta .
\end{aligned}
$$

Since $x_{t}(\cdot, t)=z(\cdot, t)$ constitutes a mild solution of (11), (14)-(17) and verifies the boundary value problem (18)-(19) in distributions, (39) is further evaluated as

$$
\begin{aligned}
\dot{\tilde{V}}(t)= & \lambda_{1} \theta z(1, t) \operatorname{sign}(x(1, t))+W_{1} \theta x(1, t) z(1, t) \\
& +\theta \int_{0}^{1} z(\eta, t) z_{\xi \xi}(\eta, t) d \eta \\
& +\theta[\dot{u}(t)+\dot{\psi}(t)] \int_{0}^{1} z(\eta, t) \delta(\eta-1) d \eta \\
= & \lambda_{1} \theta z(1, t) \operatorname{sign}(x(1, t))+W_{1} \theta x(1, t) z(1, t) \\
& +\theta \int_{0}^{1} z(\eta, t) z_{\xi \xi}(\eta, t) d \eta+\theta[\dot{u}(t)+\dot{\psi}(t)] z(1, t) .
\end{aligned}
$$

The integral term in the right hand side of (40), being integrated by parts under the homogeneous BC's (19), yields

$$
\begin{aligned}
\theta \int_{0}^{1} z z_{\xi \xi} d \eta= & \theta\left[z(1, t) z_{\xi}(1, t)-z(0, t) z_{\xi}(0, t)\right] \\
& -\theta \int_{0}^{1} z_{\xi}^{2}(\eta, t) d \eta=-\theta\left\|z_{\xi}(\cdot, t)\right\|_{0}^{2} .
\end{aligned}
$$

By substituting the closed-loop controller (11) into the last term of (40) one obtains

$$
\begin{aligned}
\theta z(1, t)[\dot{u}(t)+\dot{\psi}(t)] \\
=\theta z(1, t) \dot{u}(t)+\theta z(1, t) \dot{\psi}(t) \\
=\theta \lambda_{1} z(1, t) \operatorname{sign} x(1, t)-\theta \lambda_{2} z(1, t) \operatorname{sign} z(1, t) \\
\quad-\theta W_{1} z(1, t) x(1, t)-\theta W_{2} z^{2}(1, t)+\theta z(1, t) \dot{\psi}(t),
\end{aligned}
$$

and the next simplification

$$
\begin{aligned}
\dot{\tilde{V}}(t)= & -\lambda_{2} \theta|z(1, t)|-\theta W_{2} z^{2}(1, t)-\theta\left\|z_{\xi}(\cdot, t)\right\|_{0}^{2} \\
& +\theta z(1, t) \dot{\psi}(t)
\end{aligned}
$$

of the time derivative (40) is then obtained. Due to the upper bound (6) on the time derivative of the boundary disturbance, one obtains

$|\theta z(1, t) \dot{\psi}(t)| \leq \theta M|z(1, t)|$,

and, by (43), relation (42) is further manipulated to

$$
\begin{aligned}
\dot{\tilde{V}}(t) \leq & -\theta\left(\lambda_{2}-M\right)|z(1, t)|-\theta W_{2} z^{2}(1, t) \\
& -\theta\left\|z_{\xi}(\cdot, t)\right\|_{0}^{2} .
\end{aligned}
$$

Due to (12) and (44), the Lyapunov functional $\tilde{V}(t)$, being computed along the solutions of the closed-loop system, is a nonincreasing function of time, hence the domain $\mathscr{D}_{R}^{\tilde{V}}$, given in (33) and specified with $p=x$ and $h=x_{t}$ and an arbitrary $R \geq \tilde{V}(0)$, is invariant for the $x(\cdot, t)$ trajectories. Thus, the subsequent analysis will take into account that those solutions stay in the domain $\mathscr{D}_{R}^{\tilde{V}}$ forever.
Now consider the "augmented" functional

$$
\begin{aligned}
\tilde{V}_{R}(t)= & \tilde{V}(t)+\frac{1}{2} \kappa_{R} \theta W_{2} x^{2}(1, t)+\kappa_{R} \int_{0}^{1} x(1, t) x_{t}(\xi, t) d \xi \\
= & \lambda_{1} \theta|x(1, t)|+\frac{1}{2} \theta W_{1} x^{2}(1, t)+\frac{1}{2}\left\|x_{t}(\cdot, t)\right\|_{0}^{2} \\
& +\frac{1}{2} \kappa_{R} \theta W_{2} x^{2}(1, t)+\kappa_{R} \int_{0}^{1} x(1, t) x_{t}(\xi, t) d \xi
\end{aligned}
$$

where $\kappa_{R}$ is a sufficiently small positive constant to subsequently be specified. Note that the integral term in the right-hand side of (45) is sign-indefinite, and therefore, the positive-definiteness of the Lyapunov functional (45) has to be analyzed.

By Lemma 3 specified with $p=x$ and $h=x_{t}$, in the corresponding domain $D_{R}^{\tilde{V}}$ function $\tilde{V}_{R}$ can be lower estimated as

$$
\begin{aligned}
& \tilde{V}_{R}\left(x, x_{t}\right) \\
& \geq \lambda_{1} \theta|x(1, t)|+\frac{1}{2} \theta\left(W_{1}+\kappa_{R} W_{2}\right) x^{2}(1, t) \\
& \quad+\frac{1}{2}\left\|x_{t}(\cdot, t)\right\|_{0}^{2}-\frac{\kappa_{R}}{2}\left[\frac{R}{\lambda_{1} \theta}|x(1, t)|+\left\|x_{t}(\cdot, t)\right\|_{0}^{2}\right] \\
& =\left(\lambda_{1} \theta-\frac{\kappa_{R} R}{2 \lambda_{1} \theta}\right)|x(1, t)| \\
& \quad+\frac{1}{2} \theta\left(W_{1}+\kappa_{R} W_{2}\right) x^{2}(1, t)+\frac{1}{2}\left(1-\kappa_{R}\right)\left\|x_{t}(\cdot, t)\right\|_{0}^{2} .
\end{aligned}
$$

Let us specify $\kappa_{R}>0$ such that

$\kappa_{R}<\min \left\{\frac{2 \lambda_{1}^{2} \theta^{2}}{R}, 1\right\}$.

Then, it follows from (46), (47) that the augmented functional (45) is lower estimated by functional (23) as

$\tilde{V}_{R}\left(x, x_{t}\right) \geq \mu \tilde{V}\left(x, x_{t}\right)$

$\mu=\min \left\{1-\frac{\kappa_{R} R}{2 \lambda_{1}^{2} \theta^{2}}, \frac{W_{1}+\kappa_{R} W_{2}}{W_{1}},\left(1-\kappa_{R}\right)\right\}$.

It means that along with (23), the functional $\tilde{V}_{R}$ is positive definite on the solutions $x(\cdot, t)$ of the boundary-value problem (8)-(11) within the invariant set $\mathcal{D}_{R}^{\tilde{V}}$ of Lemma 3 specified with $p=x$ and $h=x_{t}$. Let us now evaluate the time derivative of $\tilde{V}_{R}(t)$ :

$$
\begin{aligned}
\dot{\tilde{V}}_{R}= & \dot{\tilde{V}}+\kappa_{R} \theta W_{2} x(1, t) x_{t}(1, t)+\kappa_{R} \frac{d}{d t} \int_{0}^{1} x(1, t) x_{t}(\xi, t) d \xi \\
= & \dot{\tilde{V}}+\kappa_{R} \theta W_{2} x(1, t) z(1, t)+\kappa_{R} \int_{0}^{1} z(1, t) z(\xi, t) d \xi \\
& +\kappa_{R} \int_{0}^{1} x(1, t) z_{t}(\xi, t) d \xi
\end{aligned}
$$

where $z(\cdot, t)=x_{t}(\cdot, t)$ as before. The magnitude of the first integral term in the right hand side of (50) is upper-estimated by

$$
\left|\kappa_{R} \int_{0}^{1} z(1, t) z(\xi, t) d \xi\right| \leq \kappa_{R}|z(1, t)| \int_{0}^{1}|z(\xi, t)| d \xi .
$$

If applied to the spatial domain of the unit length, the CauchySchwartz inequality ensures that

$\int_{0}^{1}|z(\xi, t)| d \xi \leq\|z(\cdot, t)\|_{0}$. 
Thus, by utilizing the second inequality of(35) specified with $h=z$ it follows from (51) and (52) that

$$
\begin{aligned}
\left|\kappa_{R} \int_{0}^{1} z(1, t) z(\xi, t) d \xi\right| & \leq \kappa_{R}|z(1, t)|\|z(\cdot, t)\|_{0} \\
& \leq \sqrt{2 R} \kappa_{R}|z(1, t)| .
\end{aligned}
$$

By straightforward integration along the weak solutions of the closed-loop system (18)-(19), driven by (11), the last integral term in (50) can further be manipulated to

$$
\begin{aligned}
\kappa_{R} x(1, t) \int_{0}^{1} z_{t}(\xi, t) d \xi \\
=\kappa_{R} x(1, t) \int_{0}^{1}\left\{\theta z_{\xi \xi}(\xi, t)+\theta[\dot{u}(t)+\dot{\psi}(t)] \delta(x-1)\right\} d \xi \\
=\kappa_{R} \theta x(1, t)\left[z_{\xi}(1, t)-z_{\xi}(0, t)\right] \\
\quad+\kappa_{R} \theta x(1, t)[\dot{u}(t)+\dot{\psi}(t)]=-\kappa_{R} \theta \lambda_{1}|x(1, t)| \\
\quad-\kappa_{R} \theta \lambda_{2} x(1, t) \operatorname{sign} z(1, t)-\kappa_{R} \theta W_{1} x^{2}(1, t) \\
\quad-\kappa_{R} \theta W_{2} x(1, t) z(1, t)+\kappa_{R} \theta x(1, t) \dot{\psi}(t) .
\end{aligned}
$$

By virtue of Assumption 1, the following estimates

$\left|\kappa_{R} \theta \lambda_{2} x(1, t) \operatorname{sign} z(1, t)\right| \leq \kappa_{R} \theta \lambda_{2}|x(1, t)|$

$\left|\kappa_{R} \theta x(1, t) \dot{\psi}(t)\right| \leq \kappa_{R} \theta M|x(1, t)|$

hold for the corresponding terms in (54). Employing (44) and (51)-(56), the time derivative (50) is finally manipulated to

$$
\begin{aligned}
\dot{\tilde{V}}_{R}(t) \leq & -\theta\left(\lambda_{2}-M-\frac{\kappa_{R} \sqrt{2 R}}{\theta}\right)|z(1, t)|-\theta W_{2} z^{2}(1, t) \\
& -\theta\left\|z_{\xi}(\cdot, t)\right\|_{0}^{2}-\kappa_{R} \theta\left[\left(\lambda_{1}-\lambda_{2}\right)-M\right]|x(1, t)| \\
& -\kappa_{R} \theta W_{1} x^{2}(1, t) .
\end{aligned}
$$

It is clear that all the terms appearing in the right-hand side of (57) are nonpositive provided that the tuning condition (12), imposed on the controller parameters, hold and, in place of (47), the next more restrictive condition on the coefficient $\kappa_{R}$ is additionally satisfied:

$\kappa_{R}<\min \left\{\frac{2 \lambda_{1}^{2} \theta^{2}}{R}, 1, \frac{\theta\left(\lambda_{2}-M\right)}{\sqrt{2 R}}\right\}$.

By Lemma 1, the following inequality

$z^{2}(1, t)+\left\|z_{\xi}(\cdot, t)\right\|_{0}^{2} \geq \frac{1}{2}\|z(\cdot, t)\|_{0}^{2}$

holds. In light of the above, the next estimate

$$
-\theta W_{2} z^{2}(1, t)-\theta\left\|z_{\xi}(\cdot, t)\right\|_{0}^{2} \leq-\theta \gamma_{1}\|z(\cdot, t)\|_{0}^{2}
$$

is obtained with $\gamma_{1}=\frac{1}{2} \min \left\{W_{2}, 1\right\}$. Relation (57) can then be manipulated to

$$
\begin{aligned}
\dot{\tilde{V}}_{R}(t) \leq & -\theta\left(\lambda_{2}-M-\frac{\kappa_{R} \sqrt{2 R}}{\theta}\right)|z(1, t)|-\theta \gamma_{1}\|z(\cdot, t)\|_{0}^{2} \\
& -\kappa_{R} \theta\left[\left(\lambda_{1}-\lambda_{2}\right)-M\right]|x(1, t)|-\kappa_{R} \theta W_{1} x^{2}(1, t) \\
\leq & -\gamma_{2}\left(|x(1, t)|+x^{2}(1, t)+\|z(\cdot, t)\|_{0}^{2}\right)
\end{aligned}
$$

where $\gamma_{2}=\theta \min \left\{\kappa_{R}\left[\left(\lambda_{1}-\lambda_{2}\right)-M\right], \kappa_{R} W_{1}, \gamma_{1}\right\}$.

On the other hand, (46), represented in terms of $z=x_{t}$, is readily estimated as

$\tilde{V}_{R}(t) \geq \gamma_{3}\left(|x(1, t)|+x^{2}(1, t)+\|z(\cdot, t)\|_{0}^{2}\right)$, where

$\gamma_{3}=\min \left\{\left(\lambda_{1} \theta-\frac{\kappa_{R} R}{2 \lambda_{1} \theta}\right), \frac{1}{2} \theta\left(W_{1}+\kappa_{R} W_{2}\right), \frac{1}{2}\left(1-\kappa_{R}\right)\right\}$.

Relations (61) and (62), coupled together, result in

$\dot{\tilde{V}}_{R}(t) \leq-\frac{\gamma_{2}}{\gamma_{3}} \tilde{V}_{R}(t)$

that establishes the exponential decay of $\tilde{V}_{R}(t)$, initialized within the invariant set $\mathscr{D}_{R}^{\tilde{V}}$ in (33).

To complete the proof it remains to note that due to the upper estimate (48) of the functional $\tilde{V}(t)$ by the functional $\tilde{V}_{R}(t)$, it follows that while being computed on the solutions $x(\cdot, t)$ of the closed-loop system (8)-(11), which are initialized within (33), the functional $\tilde{V}(t)$ exponentially decays, too. By applying Lemma 2, the local asymptotic stability of (8)-(11) is then established in $H^{2}(0,1)$ for the initial set $(33)$. Since $(33)$ can be specified with an arbitrarily large $R>0$, thus capturing an arbitrarily large initial domain, whereas the tuning conditions (13) do not depend on $R$, the global asymptotic stability in the $H^{2}(0,1)$-space is then concluded. Theorem 1 is thus proved.

\section{Simulations}

Consider the perturbed heat equation (2) with diffusivity $\theta=$ 10 and homogeneous Neumann-type BC's as in (4). In the TEST 1, the disturbance $\psi(t)$ is set to

$\psi(t)=t+\sin (\pi t)$.

The magnitude of the disturbance time derivative $\psi_{t}$ can be upper-estimated as $M=1+\pi$, as required by (6). The constant reference value $Q^{r}=30$ has been considered, whereas the initial conditions have been set to $Q^{0}(\xi)=10+5 \cos (4 \pi \xi)$.

For solving the closed-loop PDE, a standard finite-difference approximation method is used by discretizing the spatial solution domain $\xi \in[0,1]$ into a finite number of $N$ uniformly spaced solution nodes $\xi_{i}=i h, h=1 /(N+1), i=1,2, \ldots, N$. The value $N=30$ has been used. The resulting 30 -th order discretized system is solved by fixed-step Euler method with step $T_{s}=10^{-4}$.

Controller (11) has been implemented with the parameters $\lambda_{1}=10, \lambda_{2}=5, W_{1}=W_{2}=5$ which are selected in accordance with (12). The upper plot of Fig. 1 depicts the spatiotemporal profile of the solution $Q(\xi, t)$. The attainment of the prescribed constant reference confirms the expected convergence properties. The lower plot of Fig. 1 shows that the quantity $x_{\xi}(1, t)=$ $u(t)+\psi(t)$ escapes to zero, i.e., the control input $u(t)$ approaches the sign-reversed disturbance $-\psi(t)$ as $t \rightarrow \infty$. Actually, this property is theoretically verified by coupling Theorem 1 to the Agmon's inequality as the latter ensures the spatially point-wise decaying of $x_{\xi}(\xi, t)$ to zero provided that $\lim _{t \rightarrow \infty}\|x(\cdot, t)\|_{2}=0$.

Fig. 2 reports the time evolution of the $H^{2}$ norm $\|x(\cdot, t)\|_{2}$ of the regulation error in the TEST 1 , which asymptotically vanishes according to the Theorem 1.

In TEST 2, a different disturbance profile is considered of the form $\psi(t)=t+\sin (\pi t)+\psi_{R}(t)$, with $\psi_{R}(t)$ being selected as a bounded uniformly distributed random signal with the maximal magnitude value 3 . The random component is switched off at $t \geq 7$ to better appreciate its effects on the accuracy of the control system. In TEST 3, a chirp disturbance signal of the form $\psi(t)=t+\sin (2 \pi f(t) t)$ is adopted, with the time varying frequency $f(t)=0.1+4.99 t$ yielding a linear-in-time increase of the frequency from the initial value of $0.1 \mathrm{~Hz}$ (at the initial time $t=0$ ) up to the value of $50 \mathrm{~Hz}$ at the final simulation time $t=10$. The random and chirp entries of the disturbance were not considered in the evaluation of the bounding constant $M$ in (6), 

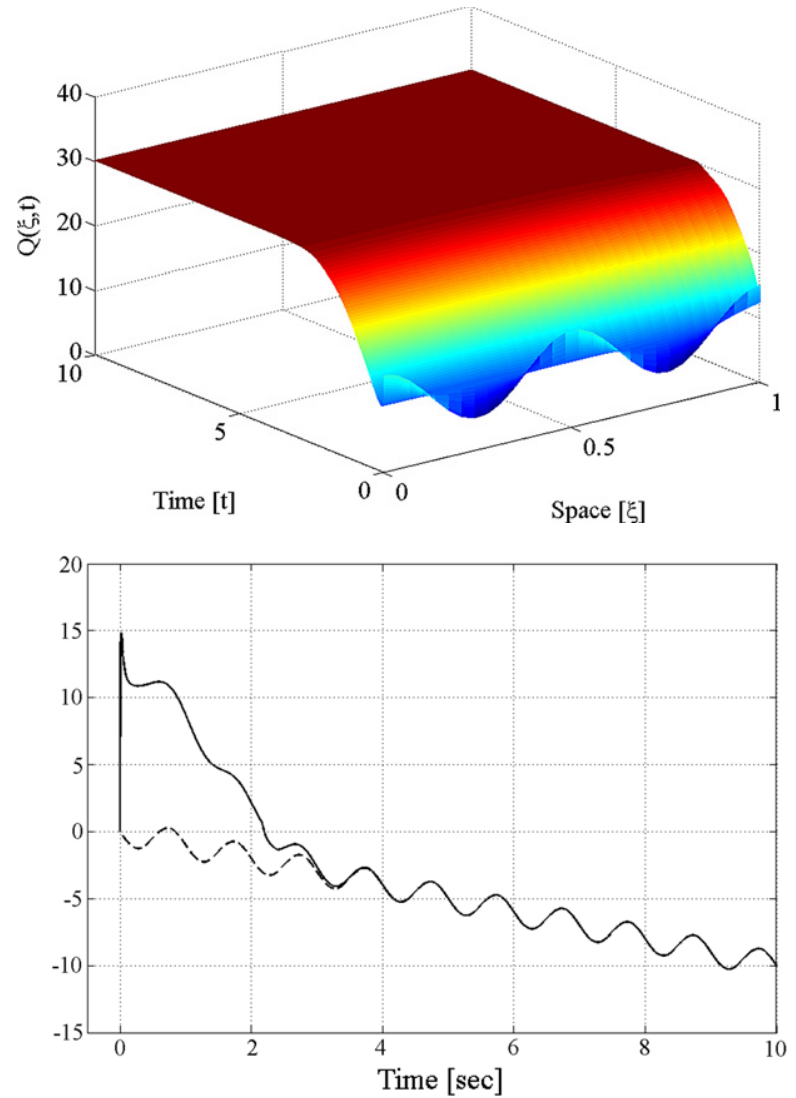

Fig. 1. TEST 1. Upper plot: the solution $Q(\xi, t)$. Lower plot: the control input $u(t)$ (solid line) and the disturbance signal $-\psi(t)$ (dashed line).

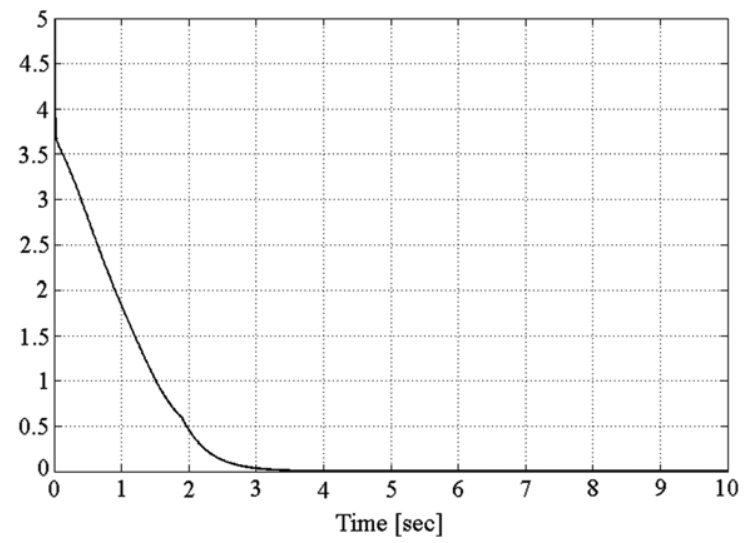

Fig. 2. TEST 1 . The $H^{2}$ error norm $\|x(\cdot, t)\|_{2}$.

and for the sake of comparison the same controller gains of TEST 1 were used in all tests. The steady-state temporal evolutions of the temperature error $H^{2}$-norm $\|x(\cdot, t)\|_{2}$ in the three tests are shown in the Fig. 3. Satisfactory steady state accuracy of the closed-loop system is concluded from Fig. 3 even in the cases of random and chirp external disturbances.

\section{Conclusions}

Using a dynamic version of a second-order sliding mode control algorithm, the problem of the boundary global asymptotic stabilization of an uncertain heat process is solved in the presence of a persistent smooth disturbance, which is generally speaking unbounded and of an arbitrary shape. The proposed control law requires boundary sensing and is synthesized by
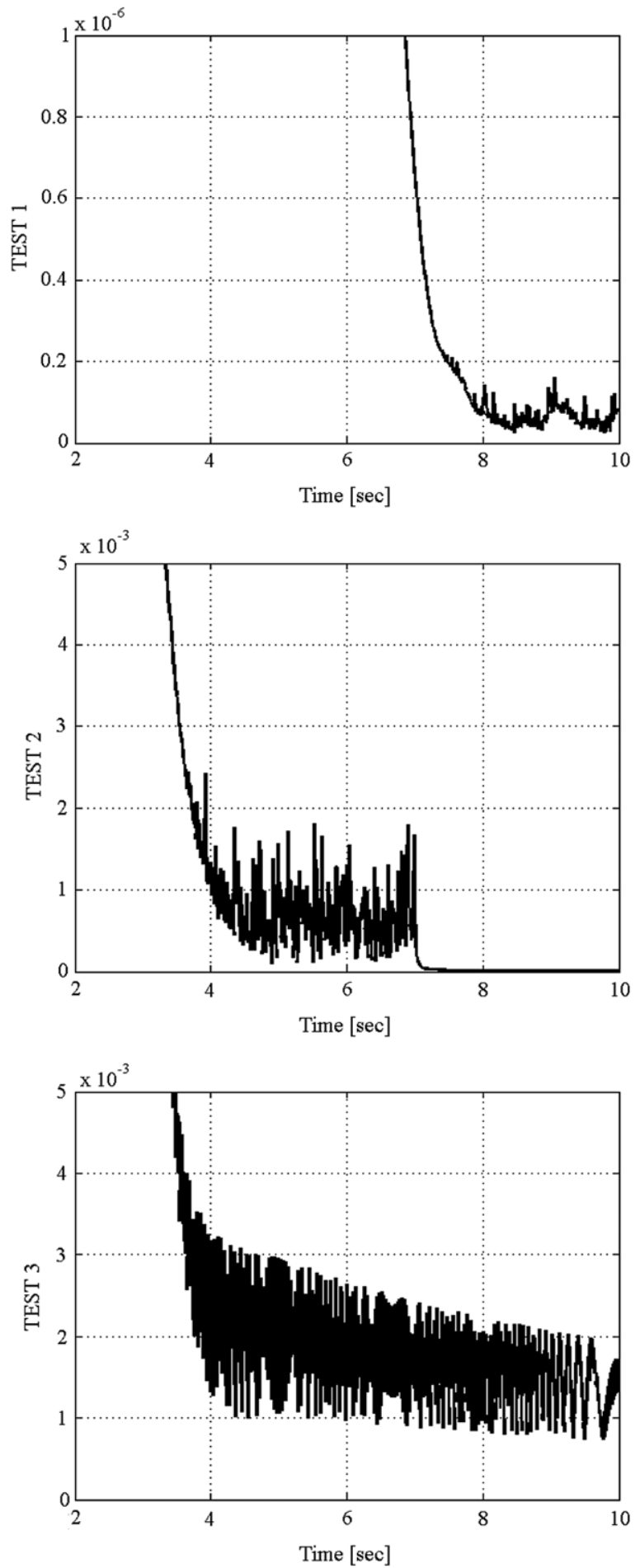

Fig. 3. Steady-state $H^{2}$ error norm $\|x(\cdot, t)\|_{2}$ in TEST 1 (upper plot), TEST 2 (central plot) and TEST 3 (lower plot).

passing a certain discontinuous signal through an integrator. The resulting control signal is therefore continuous, and the chattering phenomenon is thus attenuated. Along with this, the proposed infinite-dimensional treatment retains robustness features against non-vanishing matched disturbances similar to those possessed by its finite-dimensional counterpart. Finite-time convergence of the proposed algorithm, which would be the case if confined to a finite dimensional treatment, cannot be proved using the proposed Lyapunov functional, and it remains among other actual problems to be tackled in the future within the present framework. 


\section{References}

Boskovic, D. M., Krstic, M., \& Liu, W. (2001). Boundary control of an unstable heat equation via measurement of domain-averaged temperature. IEEE Transactions on Automatic Control, 46, 2022-2028.

Butkovskiy, A. G. (1982). Green's functions and transfer functions handbook Chichester: Ellis Horwood Ltd.

Christofides, P. D. (2001). Systems \& control: foundations E' applications series: Vol. 272. Nonlinear and robust control of PDE systems. Boston: Birkäuser.

Curtain, R., \& Zwart, H. (1995). Texts in applied mathematics, An introduction to infinite-dimensional linear systems theory. Springer.

Filippov, A. F. (1988). Differential equations with discontinuous right-hand side. Dordrecht, The Netherlands: Kluwer.

Fridman, E., \& Orlov, Y. (2009). An LMI approach to $H_{\infty}$ boundary control of semilinear parabolic and hyperbolic systems. Automatica, 45, 2060-2066.

Krstic, M., \& Smyshlyaev, A. (2008a). Adaptive boundary control for unstable parabolic PDEs. Part I. Lyapunov design. IEEE Transactions on Automatic Control, $53,1575-1591$.

Krstic, M., \& Smyshlyaev, A. (2008b). Adaptive control of PDEs. Annual Reviews in Control, 32, 149-160.

Levant, A. (1993). Sliding order and sliding accuracy in sliding mode control. International Journal of Control, 58, 1247-1263.

Levaggi, L. (2002). Sliding modes in Banach spaces. Differential and Integral Equations, 15, 167-189.

Orlov, Y. (2000). Discontinuous unit feedback control of uncertain infinitedimensional systems. IEEE Transactions on Automatic Control, 45, 834-843.

Orlov, Y. (2005). Finite-time stability and robust control synthesis of uncertain switched systems. SIAM Journal on Control and Optimization, 43, 1253-1271.

Orlov, Y. (2009). Communications and control engineering series, Discontinuous systems Lyapunov analysis and robust synthesis under uncertainty conditions. Berlin: Springer-Verlag.

Orlov, Y., Liu, Y., \& Christofides, P. D. (2004). Robust stabilization of infinitedimensional systems using sliding-mode output feedback control. International Journal of Control, 77, 1115-1136. 12.

Orlov, Y., Pisano, A., \& Usai, E. (2010). Continuous state-feedback tracking of an uncertain heat diffusion process. Systems and Control Letters, 59, 754-759.

Orlov, Y., Pisano, A., \& Usai, E. (2011a). Exponential stabilization of the uncertain wave equation via distributed dynamic input extension. IEEE Transactions on Automatic Control, 56, 212-216.

Orlov, Y., Pisano, A., \& Usai, E. (2011b). Second-order sliding-mode control of the uncertain heat and wave equations. SIAM Journal on Control and Optimization, 49, 363-382.

Orlov, Y., \& Utkin, V. I. (1987). Sliding mode control in infinite-dimensional systems. Automatica, 23, 753-757.
Pazy, A. (1992). Applied mathematical sciences series, Semigroups of linear operators and applications to partial differential equations. Berlin: Springer-Verlag.

Utkin, V. I. (1983). Sliding modes in control and optimization. New York: SpringerVerlag.

Vazquez, R., \& Krstic, M. (2007). A closed-form feedback controller for stabilization of the linearized 2-D Navier-Stokes Poiseuille system. IEEE Transactions on Automatic Control, 52, 2298-2312.

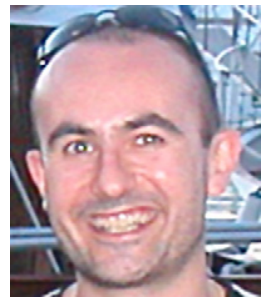

Alessandro Pisano was born in Sassari (Italy) in 1972 He graduated in Electronic Engineering in 1997 at the Department of Electrical and Electronic Engineering (DIEE) of the Cagliari University (Italy), where he received the Ph.D. degree in Electronics and Computer Science in 2000. He currently holds a permanent position as Assistant Professor at DIEE. His current research interest include sliding mode control theory and its applications to nonlinear, uncertain and/or distributed parameter systems, estimation and fault detection problems under uncertainty, fractional order systems. Within the above fields, he has authored/co-authored one book, 47 journal publications, 8 book chapters, and 65 papers in peer-reviewed conference proceedings, and is the holder of 3 patents.

Dr. Pisano is a member of IEEE and a Professional Engineer registered in Cagliari, Italy. He is Associate Editor of the Asian Journal of Control, and of the IEEE Control Systems Society Conference Editorial Board.

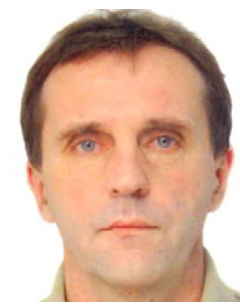

Yury Orlov received his M.S degree from the Mechanical-Mathematical Faculty of Moscow State University, in 1979, the Ph.D. degree in Physics and Mathematics from the Institute of Control Science, Moscow, in 1984, and the Dr.Sc. degree also in Physics and Mathematics from Moscow Aviation Institute, in 1990 . He has been a Full Professor of the Electronics and Telecommunication Department, Scientific Research and Advanced Studies Center of Ensenada, Mexico, since 1993. His research interests include mathematical methods in control, analysis and synthesis of nonlinear, nonsmooth, discontinuous, timedelay, distributed parameter systems, and applications to electromechanical systems. He has authored over 150 journal and conference papers in the above areas as well as three monographs, including the recent one called Discontinuous SystemsLyapunov Analysis and Robust Synthesis under Uncertainty Conditions (Springer CCE series, 2009). 